\title{
Study on Epoxy Resin-Modified Asphalt Binders with Improved Low-Temperature Performance
}

\author{
Zhiqi Luo, ${ }^{1}$ Tao Liu, ${ }^{2}$ Yintan Wu, ${ }^{2}$ Hongwei Yuan, ${ }^{1}$ Guoping Qian $\left(D,{ }^{1}\right.$ Xiantao Meng, \\ and Jun Cai $\mathbb{D}^{1}$ \\ ${ }^{1}$ Key Laboratory of Special Environment Road Engineering of Hunan Province, Changsha University of Science and Technology, \\ Changsha 410114, Hunan, China \\ ${ }^{2}$ Shenzhen Yuetong Construction Engineering Co., Ltd., Shenzhen 518000, Guangdong, China
}

Correspondence should be addressed to Guoping Qian; guopingqian@sina.com and Jun Cai; fromhscaijun@163.com

Received 31 January 2021; Revised 23 February 2021; Accepted 17 March 2021; Published 21 April 2021

Academic Editor: Shaopeng Wu

Copyright (c) 2021 Zhiqi Luo et al. This is an open access article distributed under the Creative Commons Attribution License, which permits unrestricted use, distribution, and reproduction in any medium, provided the original work is properly cited.

Epoxy resin-modified asphalt binder (ERMAB) has been wildly used in the pavement of steel bridges, while the improvement on its low temperature is still a big challenge to researchers. This paper tries to improve the low-temperature performance of ERMAB by optimizing the modifier, epoxy resin. Firstly, three epoxy resins and three amine curing agents were prepared and used for the modification of asphalt binders. Secondly, the formula and prepared methods of ERMABs were optimized and determined through compatibility, viscosity growth rate, and tensile tests. Thirdly, an overall comparison on the phase structure, thermal stability, low-temperature performance, temperature and frequency dependence, and fatigue performance of prepared ERMABs and control sample were made. Results show that polyurethane-modified epoxy resin or dimer acid-modified epoxy resin, with a suitable curing agent, can significantly improve the low-temperature performance of ERMAB, and the curing time meets the construction requirements. Compared with the control sample, the two ERMABs have basically the same rheological properties at medium temperature, but slightly worse high-temperature performance and fatigue resistance. The significance of this paper lies in proposing a feasible way to improve the low-temperature performance of ERMAB.

\section{Introduction}

Epoxy resin asphalt concrete is one of the mainstream construction forms for steel bridge deck paving. Compared with grouting asphalt concrete and SMA asphalt concrete, epoxy resin asphalt concrete, made from epoxy resin modified asphalt binder (ERMAB) and aggregates, possesses better stiffness, strength, impact resistance, and abrasion resistance and is widely employed in pavement of cross-river bridges at home and abroad [1-3]. Therefore, continuous efforts have been made to explore high-performance ERMAB.

Cong et al. [4] studied the effect of epoxy resin on improving the rheological properties of matrix asphalt binder and found that epoxy resin can not only significantly improve its high-temperature performance but also reduce its creep behavior and enhance its recovery ability. Yu et al.
[5] further studied the improvement effect of epoxy resin on SBS-modified asphalt binder from the aspects of viscosity growth, microscopic morphology, high-temperature performance, and mechanical properties. Their studies have proved that the modified asphalt binder with a suitable formula can meet the requirements of construction specifications, and its mechanical properties and rutting resistance have been significantly improved. Kang et al. [6] further compared the rheological properties of ERMAB, matrix asphalt binder, and SBS-modified asphalt binder and confirmed the above research results. Similarly, the followup studies of Yin and Cong $[7,8]$ also confirmed the conclusions of the abovementioned researchers and the feasibility that ERMAB could be applied to pavement construction of steel bridges. Gong et al. [9] studied the comprehensive performance of ERMAB mixed with waste rubber powder and Sasobit and concluded that the addition 
of Sasobit could offset the negative effects of viscosity increase caused by the addition of waste rubber powder and reduce average particle size and continuous area fraction of waste rubber powder. Jiang et al. [10] studied the micromorphology and macromorphology changes of epoxy asphalt binder modified by brominated SBS, explained the curing principle of the new modifier, and considered the epoxy asphalt binder modified by brominated SBS as an environmental-friendly material. In the study of Xin et al. [11], lignin and tung oil was used to prepare epoxy resin, which was further adopted to modify asphalt binder. The effectiveness of this new and environmental-friendly method has been affirmed by studying the performance of the above ERMAB. Qian et al. [12] studied the improvement effect of mineral fiber on epoxy asphalt binder in low-temperature crack resistance and resistance to fatigue cracking and found that the wear resistance of the modified asphalt binder was also enhanced.

It can be known from above studies that researchers have reached a consensus on the good application of ERMAB in pavement of steel bridges. Meanwhile, these results also reveal that the current research on epoxy resin asphalt binder is relatively dispersed and has not formed a system. Their research mainly focuses on the improvement of epoxy resin on the high-temperature performance and fatigue performance of asphalt binders, and the introduction of high-performance admixtures. However, there is still no systematic study on methods to improve the poor lowtemperature performance of epoxy resins and ERMABs. In fact, compared with other modified asphalt binders, ERMAB, with thermosetting epoxy resin as modifiers, exhibits worse flexibility at low temperatures, which greatly narrow its application in practical construction $[6,13,14]$. Therefore, improving the low-temperature performance of ERMAB without increasing the difficulty of construction is a problem worthy of study. This paper tries to improve the low-temperature performance of ERMAB by adopting the low-temperature-improvement epoxy resin as the modifier. Specifically, polyurethane-modified epoxy resin (U), dimer acid-modified epoxy resin (D), silicone rubber-modified epoxy resin $(\mathrm{S})$, and three self-made amine curing agents $(P$, $Q$, and $R$ ) were prepared and used to modify asphalt binder firstly. Then, the formula and prepared methods of ERMABs were further studied and determined. Finally, a comprehensive performance, including phase structure, thermal stability, low-temperature performance, dependence on temperature and frequency, and fatigue performance of prepared ERMABs were studied and compared with the control sample.

\section{Raw Materials and Experimental Methods}

\subsection{Raw Materials}

2.1.1. Epoxy Resin. In this paper, bisphenol-A-epoxy resins, E51, as the most common epoxy resin, is used as a control sample. Three types of low-temperature-modified epoxy resin are prepared, namely, polyurethane-modified epoxy resin (U), dimer acid-modified epoxy resin (D), and silicone rubber-modified epoxy resin (S). These four types of epoxy resin are used as modifiers to form modified asphalt binders (EA, UA, DA, and SA), and their performance is compared and evaluated in this paper. It should be noted here that the ratio of the E51 and curing agent used for the modified asphalt binder is the best ratio recommended by the manufacturer.

2.1.2. Curing Agent. The curing agents used in this paper are amine curing agents, which are all self-made, namely, $P, Q$, and $R$, with their curing efficiency ranging from low to high. In this part, curing time, tensile strength, and elongation at break are used to determine optimal agents. Figure 1 shows the self-made modified epoxy resin and curing agents.

2.1.3. Matrix Asphalt Binder. Relevant studies have shown that the content of asphaltene in asphalt binder is positively correlated with the compatibility of epoxy resin $[7,15]$. Therefore, considering the purpose of improving the lowtemperature performance of ERMAB in this paper, we adopt A-70 matrix asphalt binder, and its basic performance is shown in Table 1.

2.2. Preparation of ERMABs. The raw materials include epoxy resins, curing agents, and matrix asphalt binder. Firstly, we preheated the epoxy resins in an oven for 1 hour at $85^{\circ} \mathrm{C}$ and the matrix asphalt binders for 3 hours at the curing temperature. Secondly, epoxy resins and curing agents were mixed at target ratios at $500 \mathrm{rpm}$ for 5 minutes at $85^{\circ} \mathrm{C}$ in a $250 \mathrm{ml}$ beaker to obtain compound epoxy resins. Thirdly, compound epoxy resins and matrix asphalt binders were mixed at $500 \mathrm{rpm}$ for $25 \mathrm{~min}$ at the curing temperature to obtain ERMABs. It is worth noting that the epoxy resins are represented by $U, D, S$, and E51, respectively, while the prepared ERMABs is represented by UA, DA, SA, and EA to avoid confusion.

2.3. Experimental Methods. Experimental methods in this paper are different from the traditional modified asphalt binder test method, since the ratio of epoxy resin to asphalt binder should be assumed firstly so as to determine factors such as the ratio of epoxy resin to curing agent. In this experiment, firstly, in accordance with laboratory experiments routine and related references $[16,17]$, the ratio of epoxy resin to asphalt binder is assumed to be $40: 60$ by weight, based on which the type of epoxy resin, type and optimal dosage of curing agent, and the curing temperature are selected. Secondly, the optimal ratio of epoxy resin to asphalt binder is determined by changing dosages. Finally, according to the obtained formula of ERMABs, their comprehensive performance is studied and the reasons are analyzed. Also the comparisons with the performance of the control sample are made.

2.3.1. Compatibility Test. The purpose of this test is to determine appropriate epoxy resin, and observation methods are used. First, the prepared mixture of epoxy resin and hot matrix asphalt binder were stirred at $120^{\circ} \mathrm{C}$ for 5 minutes at a 


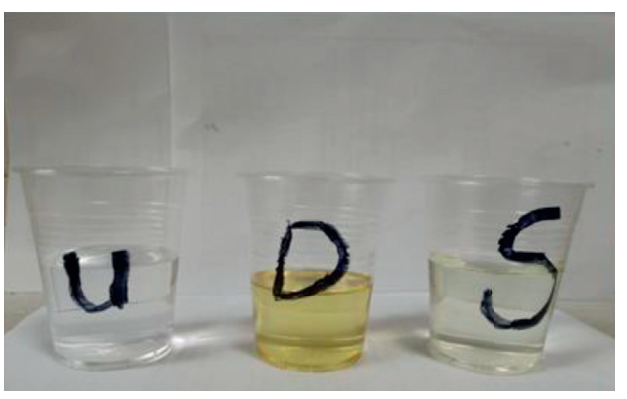

(a)

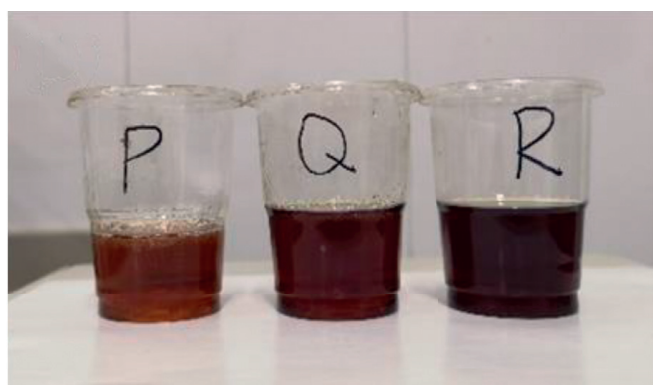

(b)

FIgURE 1: The appearance of self-made modified epoxy resins (a) and curing agents (b).

TABLE 1: Basic performance of A-70 matrix asphalt binder.

\begin{tabular}{|c|c|c|c|}
\hline \multirow{2}{*}{\multicolumn{2}{|c|}{ Items }} & \multicolumn{2}{|c|}{$\begin{array}{c}\text { A-70 matrix } \\
\text { asphalt binder }\end{array}$} \\
\hline & & Results & Index \\
\hline \multirow{3}{*}{\multicolumn{2}{|c|}{$\begin{array}{l}\text { Penetration }\left(25^{\circ} \mathrm{C}, 100 \mathrm{~g}, 5 \mathrm{~s}\right) / 0.1 \mathrm{~mm} \\
\text { Ductility }\left(15^{\circ} \mathrm{C}, 5 \mathrm{~cm} / \mathrm{min}\right) / \mathrm{cm} \\
\text { Softening point (ring and ball } \\
\text { method } /{ }^{\circ} \mathrm{C}\end{array}$}} & 74.0 & $60-80$ \\
\hline & & $>120$ & $>100$ \\
\hline & & 47.4 & $\geq 46$ \\
\hline \multicolumn{2}{|c|}{ Solubility (\%) } & 99.90 & $>99.5$ \\
\hline Residue after & Quality change (\%) & -0.1 & $\leq \pm 0.8$ \\
\hline RTFOT & Penetration ratio $(\%)$ & 73 & $\geq 61$ \\
\hline \multicolumn{2}{|c|}{$\begin{array}{l}\text { Relative density of asphalt at } 25^{\circ} \mathrm{C} / \\
\left(\mathrm{g} / \mathrm{cm}^{3}\right)\end{array}$} & 1.031 & - \\
\hline
\end{tabular}

ratio of $40: 60$. Next, we dropped some mixture with a gluetip dropper onto the prepared glass slide, covered it with a cover glass, and placed it in an oven at $120^{\circ} \mathrm{C}$ for 60 minutes. Finally, we took out the sample and studied the compatibility of the epoxy resin and the asphalt binder after it cooled to room temperature.

2.3.2. Viscosity Test. After determining the type of epoxy resin, it is necessary to select the appropriate curing agent type and dosage and curing temperature to achieve the most favorable conditions for engineering applications. The viscosity increase and mechanical properties of ERMABs during the curing processes are the main evaluation index, so the viscosity test and tensile test are employed to detect this variation.

For the viscosity test, Brookfield viscometer was adopted. The rotor was no. 27, and the speed was $50 \mathrm{r} / \mathrm{min}$. The specific test procedures were carried out in accordance with ASTM D2393 specification [18], and we recorded once every minute continuously until the viscosity reaching $5000 \mathrm{MPa} \cdot \mathrm{s}$ or the test time reaching 90 minutes. The times when the viscosity were increased to $1000 \mathrm{MPa} \cdot \mathrm{s}$ and $3000 \mathrm{MPa} \cdot \mathrm{s}$ were particularly concerned.

2.3.3. Tensile Test. The tensile test was carried out based on ASTM D638 specification [19]. Six sets of replicas were measured at the specified temperature of $23^{\circ} \mathrm{C}$ for each binder, and the evaluation indexes were tensile strength and elongation at break. The details of test samples and test processes are shown in Figure 2.
2.3.4. Microscopic Fluorescence Test. During the microscopic fluorescence test, microscopic morphology and phase structure of the epoxy resin and matrix asphalt binder within the ERMABs are observed, which could better explain the changes in its macroscopic properties. In this test, the cured ERMABs were used to make glass slide samples, which were observed in blue light in the case of 100-fold magnification with a fluorescence microscope.

2.3.5. Thermogravimetric Analysis (TGA). The TGA test mainly analyzes the thermal decomposition of the internal substances of ERMABs. In this test, the mass of the sample was $10 \mathrm{mg}$, the test temperature was in the range of $25^{\circ} \mathrm{C}$ to $900^{\circ} \mathrm{C}$, the heating rate was $10^{\circ} \mathrm{C} / \mathrm{min}$, and the protective gas was nitrogen.

2.3.6. Ductility Test. The ductility test was used to evaluate the low-temperature performance of three prepared ERMABs and the control samples. The test was carried out in accordance with ASTM D113 specification [20]. The test temperature was $5^{\circ} \mathrm{C}$ and $10^{\circ} \mathrm{C}$, the extension speed was $5 \mathrm{~cm} / \mathrm{min}$, and the evaluation index was extension length.

2.3.7. Bending Beam Rheometer (BBR). BBR was also employed to evaluate the low-temperature performance of ERMABs. The BBR test was carried out in accordance with ASTM D6373 specification [21]. The test temperature was $-6^{\circ} \mathrm{C}$, $-12^{\circ} \mathrm{C},-18^{\circ} \mathrm{C}$, and $-24^{\circ} \mathrm{C}$. The evaluation indexes were stress relaxation speed $(\mathrm{m})$ and creep stiffness modulus $(\mathrm{S})$. At least three samples of every modified asphalt binder were tested. The calculation formula of evaluation indexes is shown in

$$
\begin{gathered}
\mathrm{S}(t)=\frac{\mathrm{PL}^{3}}{4 \mathrm{bh}^{3} \delta(t)}, \\
\mathrm{m}=\frac{\mathrm{d} \lg S(t)}{\mathrm{dlg} t},
\end{gathered}
$$

where $S(t)$ refers to the creep stiffness modulus (MPa). m refers to the stress relaxation speed. $P$ refers to the constant load $(\mathrm{N})$ at the midpoint of the sample. $\mathrm{L}, \mathrm{b}$, and $\mathrm{h}$ refer to the distance between the support points of the beam, the height of the beam, and the width of the beam, respectively. 


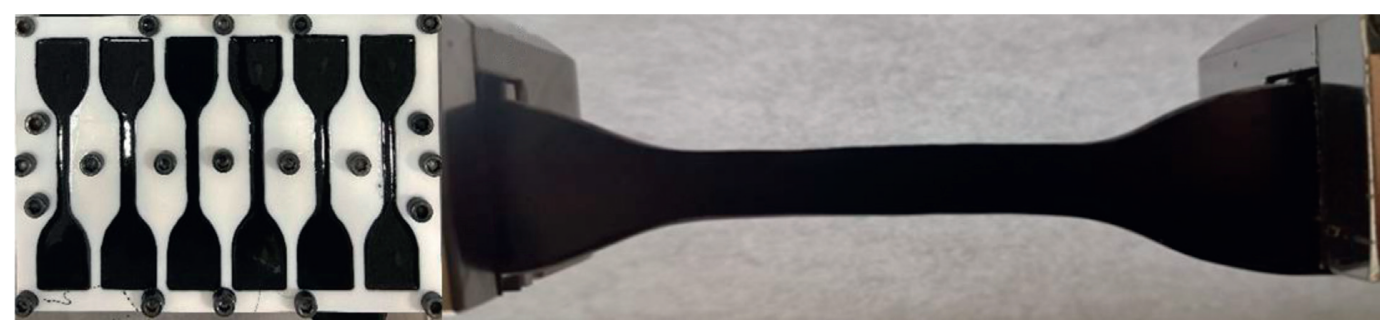

(a)

(b)

Figure 2: Dumbbell shape (a) and tensile details (b) of the test samples.

$\delta(\mathrm{t})$ refers to the deflection $(\mathrm{mm})$ of the beam midpoint under load over time.

2.3.8. Temperature and Frequency Sensitivity Tests. Temperature sensitivity test and frequency sensitivity test, respectively, evaluate the dependence of ERMABs on temperature and frequency changes, both of which are carried out by using dynamic shearing rheometer. During the temperature sweep test, the test temperature range was from $30^{\circ} \mathrm{C}$ to $90^{\circ} \mathrm{C}$, the frequency was $10 \mathrm{rad} / \mathrm{s}$, and the shear strain was $5 \%$. In the frequency sweep test, the test temperature was $15^{\circ} \mathrm{C}, 30^{\circ} \mathrm{C}, 45^{\circ} \mathrm{C}$, and $60^{\circ} \mathrm{C}$, the frequency was $0.1-100 \mathrm{~Hz}$, and the shear strain was $2 \%$. After the temperature sweep test, complex modulus $G^{*}$ and phase angle $\delta$ were employed for evaluation. After the frequency sweep test, the frequency master curve was used for evaluation, and the master curve reference temperature was $30^{\circ} \mathrm{C}$.

2.3.9. Multiple Stress Creep Recovery (MSCR). MSCR is used to evaluate the fatigue performance of ERMABs. The test was carried out in accordance with AASHTO MP19 specification, and the test temperature was $64^{\circ} \mathrm{C}$ [22]. Samples were tested for 10 cycles with pressures of $0.1 \mathrm{kPa}$ and $3.2 \mathrm{MPa}$, respectively. After the test, the relationship curve between shear strain and time, the recovery rate (R), and nonrecoverable recovery creep compliance (Jnr) in the deformation were used to analyze the results. The calculation equations of $\mathrm{R}$ and Jnr are shown in

$$
\begin{aligned}
\mathrm{R} & =\frac{\gamma_{\mathrm{p}}-\gamma_{\mathrm{nr}}}{\gamma_{\mathrm{p}}-\gamma_{0}}, \\
\mathrm{~J}_{\mathrm{nr}} & =\frac{\gamma_{\mathrm{nr}}-\gamma_{0}}{\tau},
\end{aligned}
$$

where $\gamma_{\mathrm{p}}$ refers to the peak shear strain of each loading cycle. $\gamma_{\mathrm{nr}}$ refers to the residual shear strain of each loading cycle. $\gamma_{0}$ refers to the initial shear strain of each loading cycle.

\section{Formula and Prepared Methods of ERMABs}

3.1. Epoxy Resin. Figure 3 shows the results of the compatibility test of epoxy resin E51, U, D, and S and matrix asphalt binders. It can be seen that in the test $S$ and matrix asphalt binders were obviously segregated, while $U, D$, and E51 were not. The reason is that $S$ is modified by silicone rubber. The calculated solubility parameter of silicone rubber is $7.65\left(\mathrm{~J} / \mathrm{cm}^{3}\right)^{1 / 2}$, while the solubility parameter of matrix binder is $15.68\left(\mathrm{~J} / \mathrm{cm}^{3}\right)^{1 / 2}$, and the large difference between the two leads to poor compatibility [23,24]. Therefore, $\mathrm{S}$ will no longer be used in subsequent experiments.

\subsection{Curing Agent}

3.2.1. Types of Curing Agent. As mentioned before, the optimization of curing agent $\mathrm{P}, \mathrm{Q}$, and $\mathrm{R}$ was determined by comparing viscosity growth rates, tensile strength, and elongation at break of different ERMABs.

Figure 4 shows the viscosity-time increasing curves of $\mathrm{UA}$ and DA with curing agent $\mathrm{P}, \mathrm{Q}$, and $\mathrm{R}$. In these results, the ratio of curing agent to $U$ was 1 to 9 , and the ratio of curing agent to $\mathrm{D}$ was 1 to 21 according to laboratory experience. It can be seen that the curing processes of ERMABs with $\mathrm{Q}$ and $\mathrm{R}$ were obviously faster. In terms of $\mathrm{DA}$, when the curing agent was $R$, the viscosity of the ERMAB reached $1000 \mathrm{MPa} \cdot \mathrm{s}$ after 35 minutes and $3000 \mathrm{MPa} \cdot \mathrm{s}$ after 51 minutes; when the curing agent was $\mathrm{Q}$, the viscosity reached $1000 \mathrm{MPa} \cdot \mathrm{s}$ after 46 minutes, and reached $3000 \mathrm{MPa} \cdot \mathrm{s}$ after 61 minutes; when the curing agent was $P$, the viscosity reached $1000 \mathrm{MPa} \cdot \mathrm{s}$ after 56 minutes and reached $3000 \mathrm{MPa} \cdot \mathrm{s}$ after 73 minutes. As for UA, the viscosity of ERMABs with curing agent increased even faster. When the curing agent was $\mathrm{P}$, the viscosity reached $1000 \mathrm{MPa} \cdot \mathrm{s}$ after 54 minutes and reached $3000 \mathrm{MPa} \cdot \mathrm{s}$ after 67 minutes. It is known that during the actual construction, a faster viscosity growth rate means a shorter residence time, which will cause additional difficulties, so sufficient time for construction is essential. Researchers believed that it is appropriate the viscosity of the ERMAB reaches $1000 \mathrm{MPa} \cdot \mathrm{s}$ after 50 minutes [25]. According to this specification, the comparison results show that $\mathrm{P}$ meets the requirements as a curing agent for both UA and DA.

Figure 5 shows different ERMABs have similar changes overall. In terms of tensile strength, the tensile strength of ERMABs with curing agents $\mathrm{P}$ and $\mathrm{R}$ was quite similar. In contrast, the tensile strength of that with curing agent $\mathrm{Q}$ was lower. In terms of elongation at break, the ERMAB prepared with curing agent $\mathrm{Q}$ the largest, followed by $\mathrm{P}$ and $\mathrm{R}$.

Based on the results of the viscosity test and tensile test, it can be considered that the curing agent $\mathrm{P}$ has advantages in terms of viscosity growth rate, tensile strength, and 


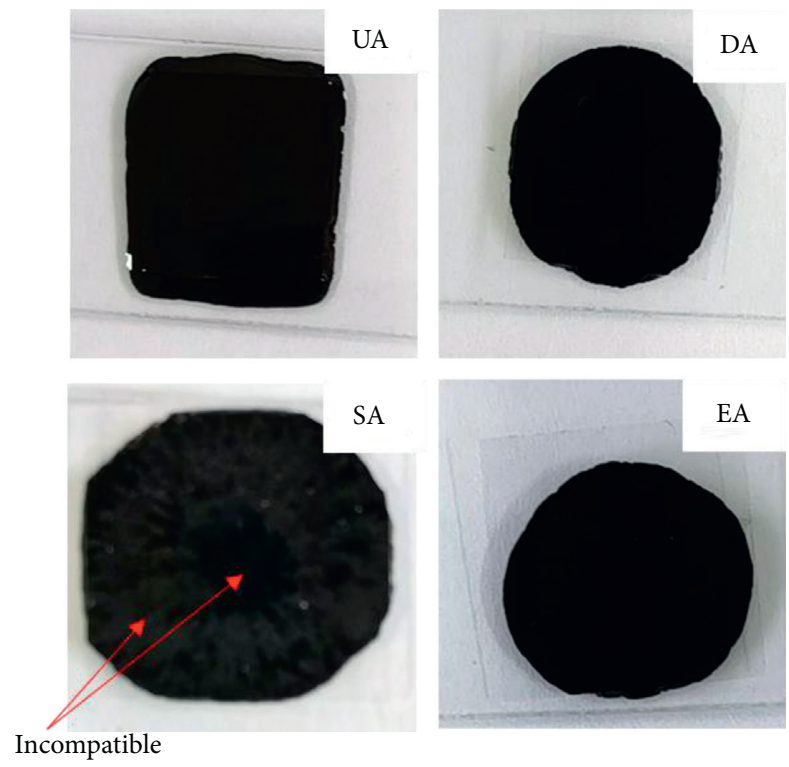

FIGURE 3: Results of compatibility test of UA, DA, SA, and EA.

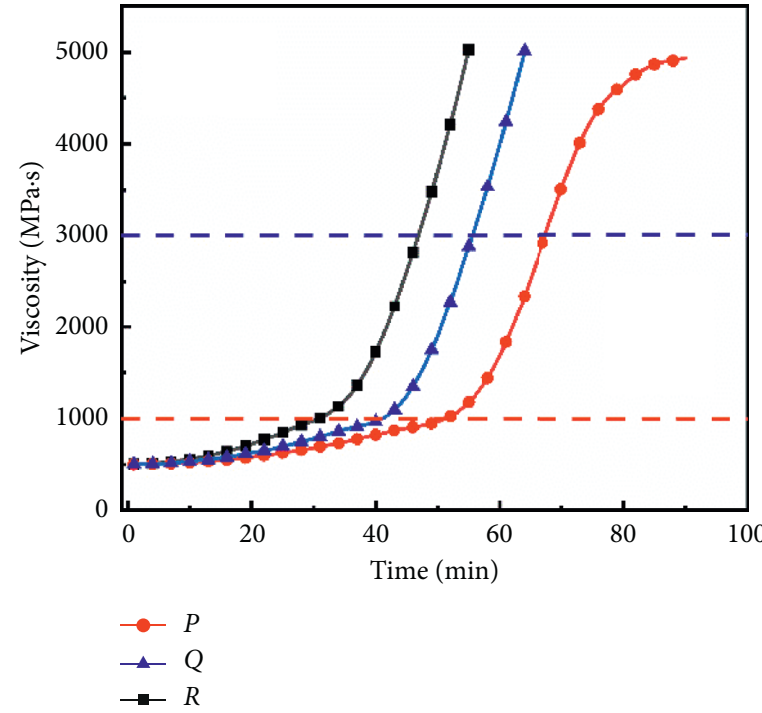

(a)

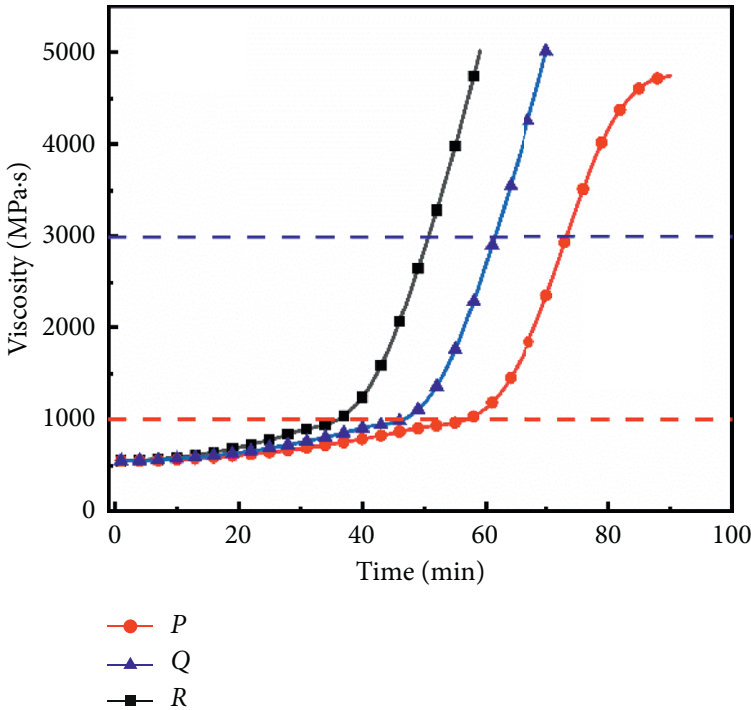

(b)

FIgURe 4: Viscosity-time curves of UA (a) and DA (b).

elongation at break. Therefore, curing agent $\mathrm{P}$ will be adopted for subsequent experiments.

3.2.2. Dosage of Curing Agent. After determining the type of curing agent, the dosage of curing agent needs to be further studied. As mentioned earlier, the experiment was carried out on the assumption that the ratio of epoxy resin to matrix asphalt binder was 40 to 60 by weight. Herein, ERMABs were prepared in the conditions that the ratio of epoxy resin $U$ to curing agent $P$ was 8 to 1,9 to 1 , and 10 to 1 , and the ratio of epoxy resin $\mathrm{D}$ to curing agent $\mathrm{P}$ was 20 to 1,21 to 1 , and 22 to 1 by weight, respectively. Finally, viscosity growth and tensile test results were analyzed.
Figure 6 shows the viscosity growth curves of ERMABs with different dosages of curing agents. Although with different epoxy resins, these two types of ERMABs have the similar curves. When the dosage of curing agent $\mathrm{P}$ was high, that is, when the ratio of $\mathrm{U}$ to $\mathrm{P}$ was 8 to 1 and $\mathrm{D}$ to $\mathrm{P}$ was 20 to 1, both ERMABs had faster curing speeds and shorter time it took to increase the viscosity to $1000 \mathrm{MPa} \cdot \mathrm{s}$ and $3000 \mathrm{MPa} \cdot \mathrm{s}$. When the dosage of curing agent $\mathrm{P}$ was low, that is, when the ratio of $\mathrm{U}$ to $\mathrm{P}$ was 10 to 1 and $\mathrm{D}$ to $\mathrm{P}$ was 22 to 1 , the viscosity of the two binders increased more slowly. Especially for DA, the viscosity could hardly increase to the relatively high value within the test time, which may decrease the mechanical properties of prepared binders. When the amount of curing agent $\mathrm{P}$ was moderate, that is, when the ratio of $\mathrm{U}$ to $\mathrm{P}$ was 9 to 


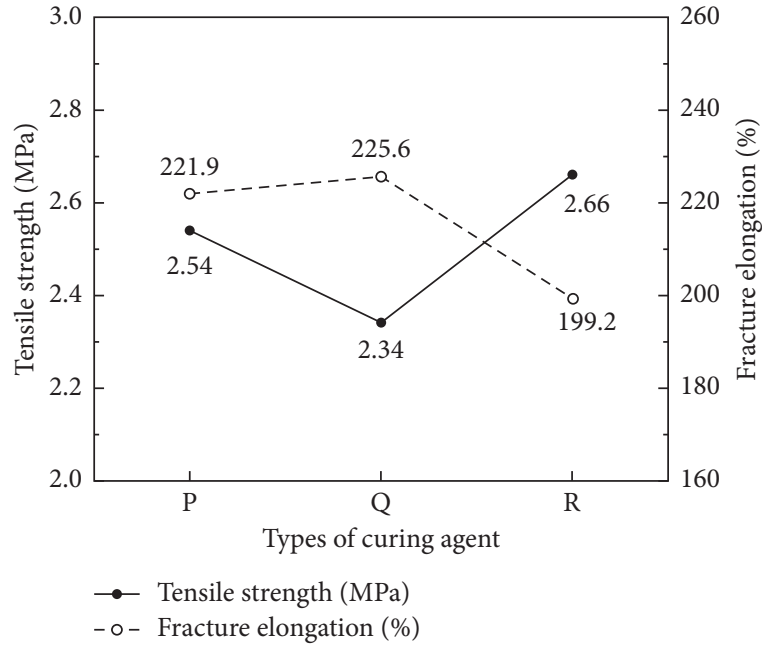

(a)

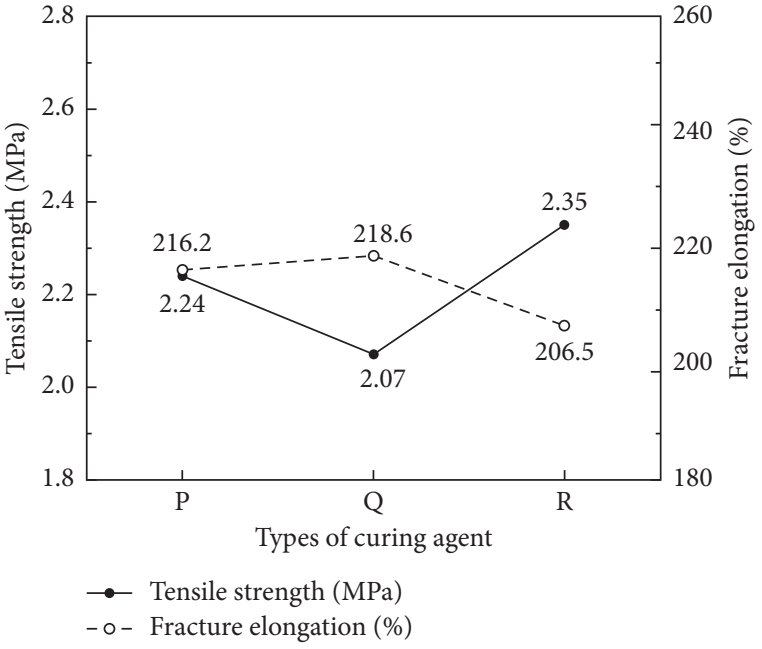

(b)

Figure 5: Tensile test results of UA (a) and DA (b) at $23^{\circ} \mathrm{C}$.

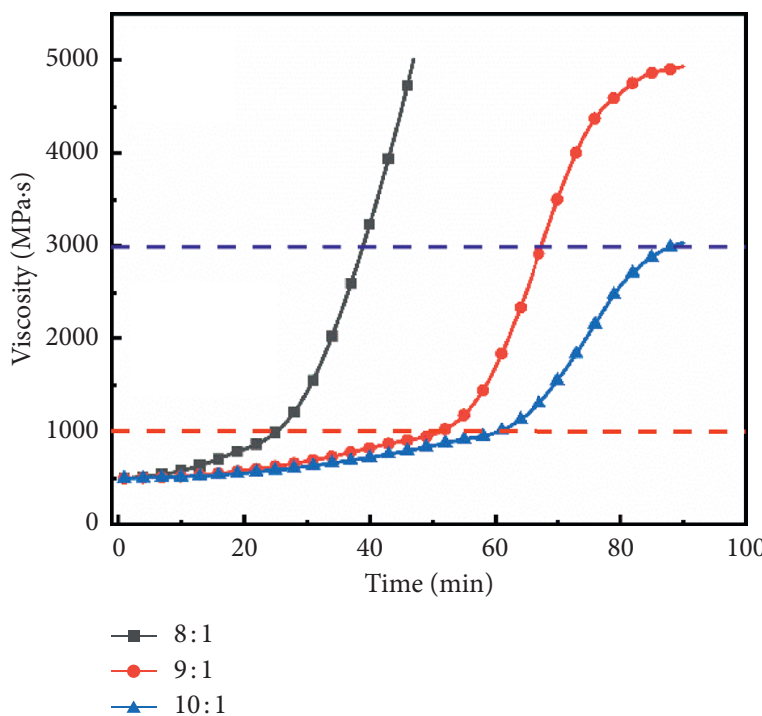

(a)

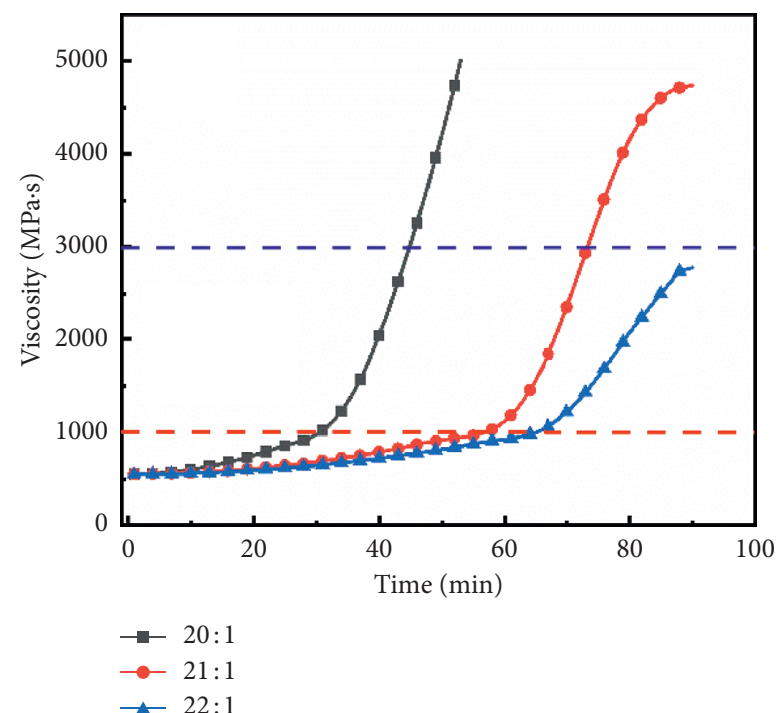

(b)

FIGURE 6: Viscosity-time curves of UA (a) and DA (b) with different dosages of curing agent.

1 and $\mathrm{D}$ to $\mathrm{P}$ was 21 to 1 , the viscosity growth rate was also moderate, and the curing time was reasonable.

The curves in Figure 7(a) show that as the dosage of curing agent gradually decreased, the tensile strength decreased, and the elongation at break gradually increased. When the ratio of $\mathrm{U}$ to $\mathrm{P}$ was changed from 8 to 1 to 9 to 1 , the tensile strength decreased by $3.8 \%$ and the elongation at break increased by $9.6 \%$. When the ratio of $\mathrm{U}$ to $\mathrm{P}$ changed from 9 to 1 to 10 to 1 , the tensile strength of the asphalt binder was significantly reduced, and the reduction rate reached $21.1 \%$, while the elongation at break increased by $4.4 \%$. Therefore, it reflected that when the dosage of curing agent is too low, the tensile strength of the ERMAB will be greatly affected. Similarly, the similar conclusion could be obtained from Figure 7(b).
Based on the above analysis, it can be concluded that the most appropriate ratios of $\mathrm{U}$ to $\mathrm{P}$ and $\mathrm{D}$ to $\mathrm{P}$ are 9 to 1 and 21 to 1 by weight, respectively.

3.3. Curing Temperature. The previous sections have studied the types and dosages of curing agents, and this section will focus on curing temperature. Curing temperature mainly affects the reaction speed of the epoxy resin and the curing agent, which determines the residence time of the ERMABs during the construction process. First, ERMABs were prepared, in the conditions that the ratio of epoxy resin to matrix asphalt binder was 40 to 60 , $\mathrm{U}$ to $\mathrm{P}$ was 9 to 1 , and $\mathrm{D}$ to $P$ was 21 to 1 by weight. Then, viscosity growth rates were 


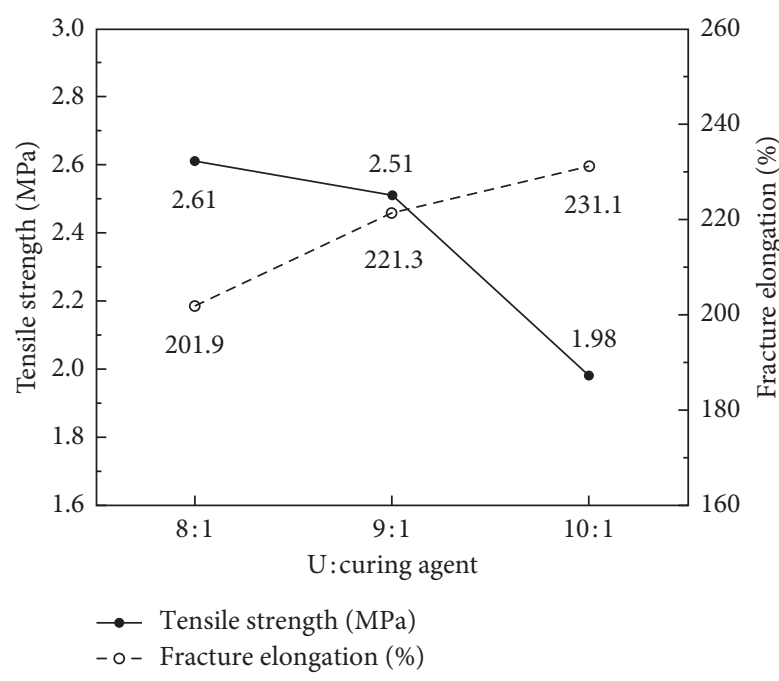

(a)

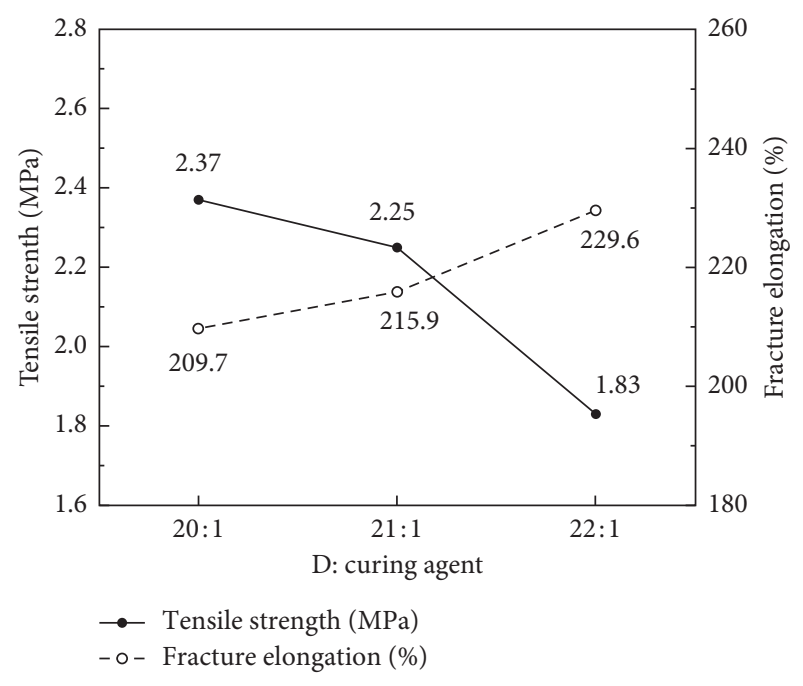

(b)

FIgURE 7: Tensile tests results of UA (a) and DA (b) with different dosages of curing agent.

studied when the curing temperature was $110^{\circ} \mathrm{C}, 120^{\circ} \mathrm{C}$, and $130^{\circ} \mathrm{C}$, respectively.

Figures 8(a) and 8(b) show similar changes of curves. When the curing temperature was $130^{\circ} \mathrm{C}$, the viscosity increases rapidly, reaching $1000 \mathrm{MP} \cdot \mathrm{s}$ within 20 minutes and $3000 \mathrm{MP} \cdot \mathrm{s}$ within 40 minutes, which does not meet the actual construction requirements. When the curing temperature dropped to $120^{\circ} \mathrm{C}$, the viscosity increase slowed down, and it took about 50 to 55 minutes to reach $1000 \mathrm{MP}$.s, and about $70 \mathrm{~min}$ to reach $3000 \mathrm{MP} \cdot \mathrm{s}$. When the curing temperature continued to decrease to $110^{\circ} \mathrm{C}$, more time was needed to reach $1000 \mathrm{MP} \cdot \mathrm{s}$, and then viscosity increased too slowly and hardly reach $3000 \mathrm{MP} \cdot \mathrm{s}$ during the test time. Therefore, $120^{\circ} \mathrm{C}$ is considered the most appropriate curing temperature in the test.

\subsection{Formula and Curing Time of ERMABs}

3.4.1. Formula. The ERMAB is composed of curing agent, epoxy resin, and matrix asphalt binder. When the ratio of epoxy resin to curing agent is determined, ratios of the two to the matrix asphalt binders should be further studied. From Sections 3.1 to 3.3, the ratio of epoxy resin to matrix asphalt binder was assumed of 40 to 60 by weight. In this section, other two ratios, namely, 30 to 70 and 50 to 50, are adopted in the test to confirm the suitable ratio.

Figures 9(a) and 9(b) show similar changes of curves. When the ratio of epoxy resin to matrix asphalt binder was $50: 50$, the viscosity increased the fastest. When the above ratio was reduced to 40 to 60 , the viscosity increased slightly slowed down and mainly decreased the reaction speed of binder within $1000 \mathrm{MP} \cdot \mathrm{s}$. When the ratio further dropped to 30 to 70 , the increase of the entire viscosity slowed down significantly, making it difficult to reach the desired high viscosity for ensuring sufficient high-temperature stability of the prepared binder.

Figure 10 shows the tensile test results of ERMABs with different ratios of epoxy resin to matrix asphalt binder.
Figure 10(a) shows that when this ratio is 30 to 70 , the overall tensile strength is the lowest and the elongation at break is the highest. When the ratio is increased to 40 to 60 , the tensile strength is increased by $14.5 \%$ and the elongation at break decreased by $7.4 \%$. When the ratio is further increased to 50 to 50 , the tensile strength decreases slightly and the elongation at break increases slightly. This indicated the proper dosage of epoxy resin is critical to the modified binder system, and either higher or lower dosage may bring negative effects on the mechanical properties of binder. Similar changes are shown in Figure 10(b).

Based on the above test results, it is believed that when the ratio of epoxy resin $U$ and $D$ to asphalt binder is 40 to 60 , the curing time of ERMABs in relatively low viscosity range is long, the tensile strength is relatively high, and the elongation at break exceeds $220 \%$. This result meets the requirements of actual construction and application [25]. Therefore, the ratio of epoxy resin to asphalt binders is 40 to 60 by weight.

3.4.2. Curing Time. Considering that this experiment adopts modified epoxy resin and self-made amine curing agents, in this section, tensile tests are used to further study the best curing time of ERMABs, with the assumption that the curing time is $2 \mathrm{~h}, 4 \mathrm{~h}$, and $6 \mathrm{~h}$, respectively.

Figure 11 shows the tensile test results of ERMABs with different curing times. In Figure 11(a), within the period of $2 \mathrm{~h}$ to $4 \mathrm{~h}$, the tensile strength increased by $30.6 \%$ and the elongation at break decreased by $4.6 \%$, which indicates that the curing reaction of the system is still going on between $2 \mathrm{~h}$ and $4 \mathrm{~h}$. Within the period of $4 \mathrm{~h}$ to $6 \mathrm{~h}$, the tensile strength hardly increased, and the elongation at break decreased by $3.3 \%$, which shows that the curing reaction is basically over. It is also worth noting that excessive curing time at relatively high temperature will also affect the low performance of modified asphalt binder for the obviously oxidative aging effect on the epoxy resin. So, it can be considered that the suitable curing time is $4 \mathrm{~h}$ from Figure 11(a). Similar conclusions can be obtained from Figure 11(b). 


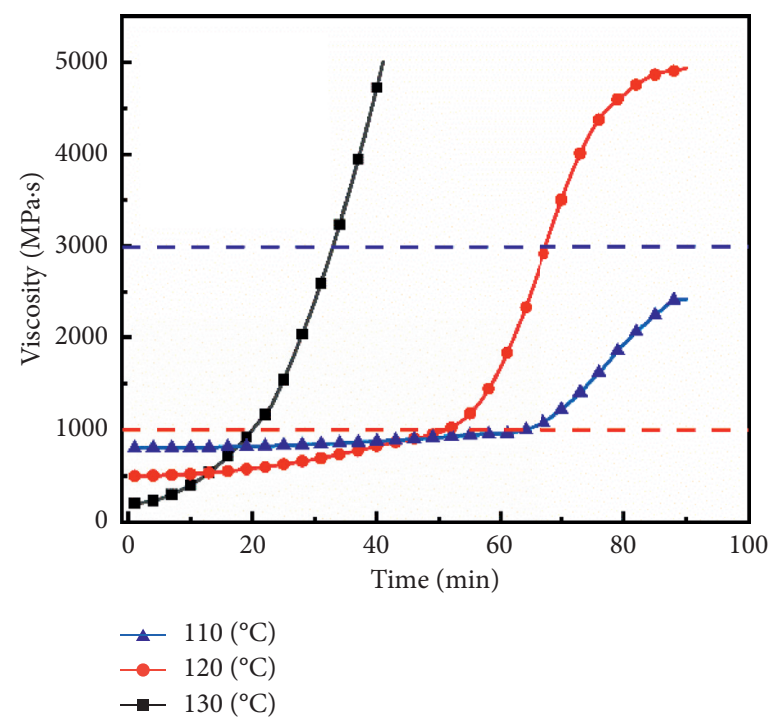

(a)

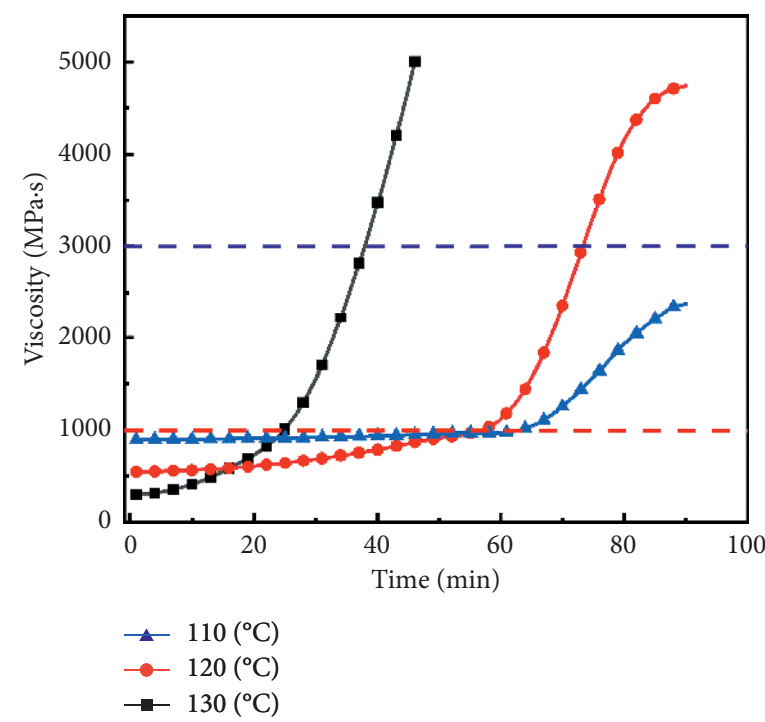

(b)

FIGURE 8: Viscosity-time curves of UA (a) and DA (b) at different curing temperatures.

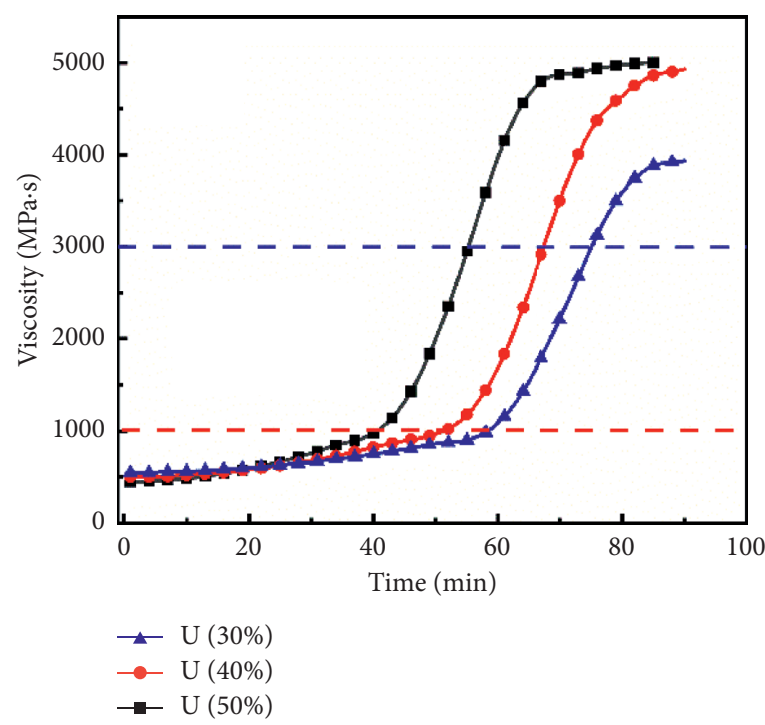

(a)

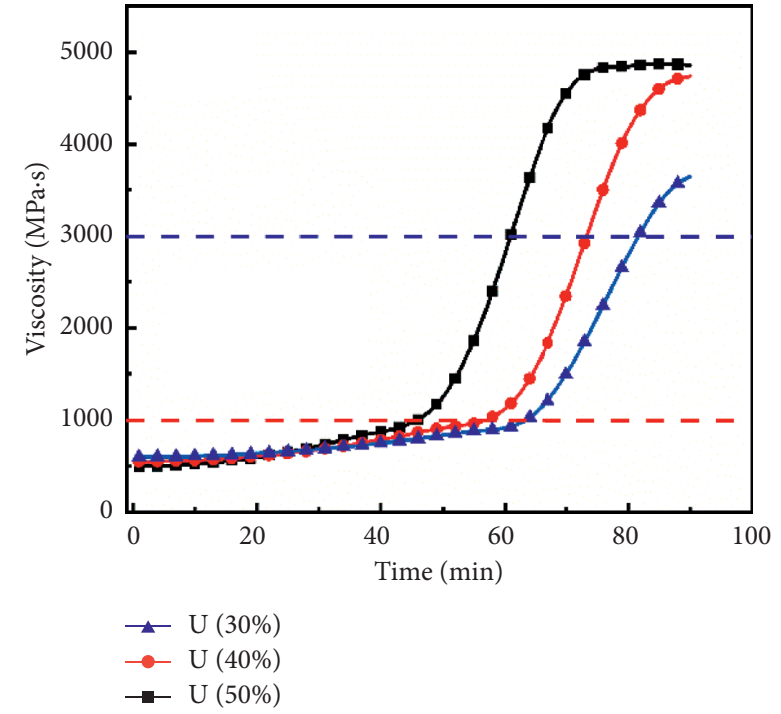

(b)

FIgURE 9: Viscosity-time curves of UA (a) and DA (b) with different ratios of epoxy resin to matrix asphalt binders.

\section{Performance of ERMABs}

In Section 3, the formula and preparation parameters of ERMABs are studied. Herein, the performance of UA and DA will be explored with the best ratio and test conditions and is compared with that of the control sample EA.

4.1. Phase Structure. In this section, phase structures of ERMABs are observed via a fluorescence microscope, as shown in Figure 12.

Figures 12(a)-12(c) are fluorescent photos of UA, in which the ratio of epoxy resin $U$ to matrix binder is 30 to 70 ,
40 to 60 , and 50 to 50 by weight, respectively. It is observed that as the proportion of epoxy resin increases, the matrix asphalt binder phase, the black part, gradually decreases, while the epoxy resin phase, the green part, gradually increases. When the ratio increases from $30: 70$ to $40: 60$, although the matrix asphalt binder phase is gradually decreasing, it is still the continuous phase, but when the ratio continues to increase to $50: 50$, the epoxy resin becomes the continuous phase and the matrix asphalt binder becomes dispersed phase. Therefore, $40: 60$ is the critical ratio for epoxy resin and matrix asphalt binder in the modified system, where the epoxy resin wraps the matrix asphalt binder and closes to the point of continuous phase reversal. 


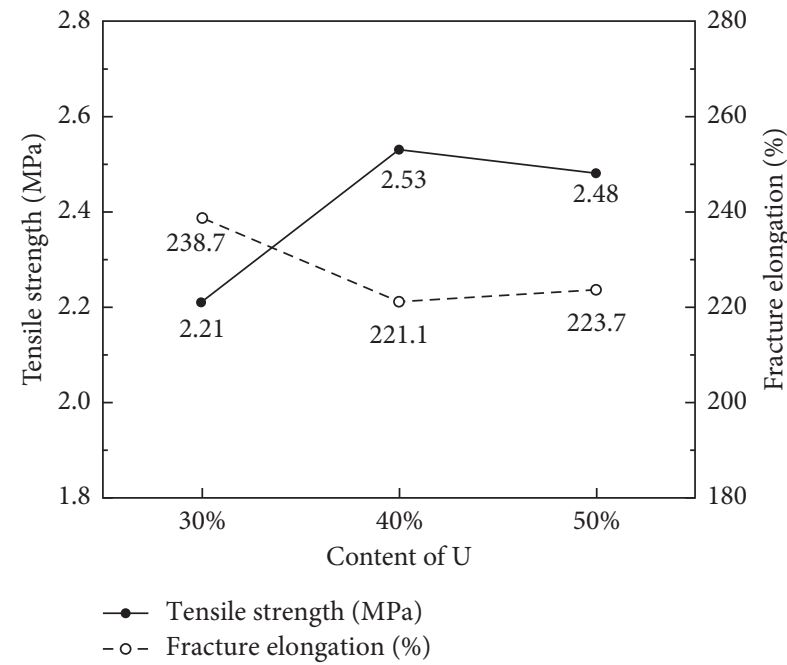

(a)

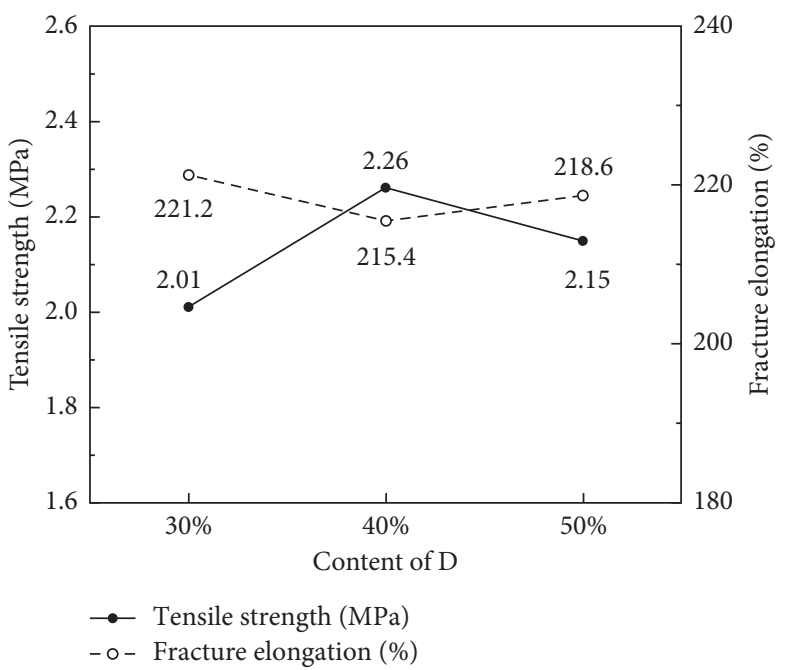

(b)

Figure 10: Tensile test results of UA (a) and DA (b) with different ratios of epoxy resin to matrix asphalt binders.

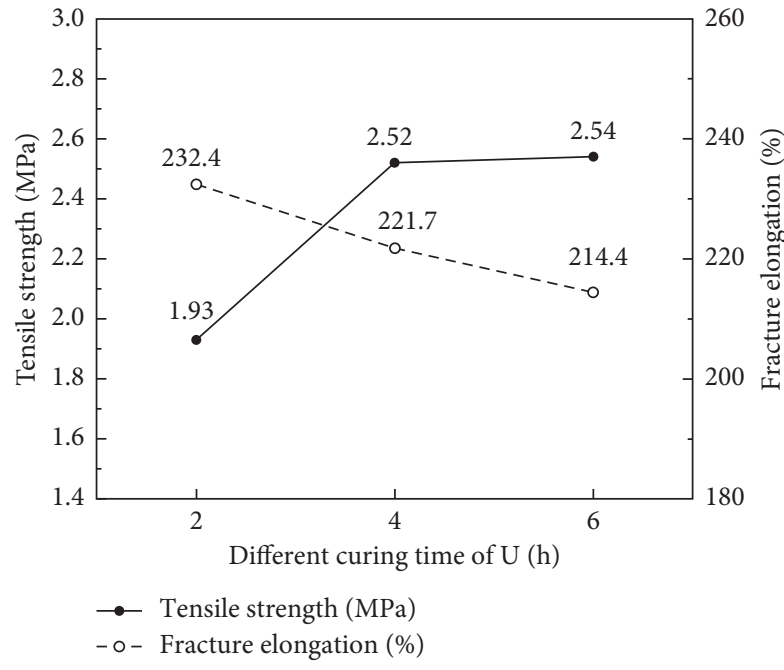

(a)

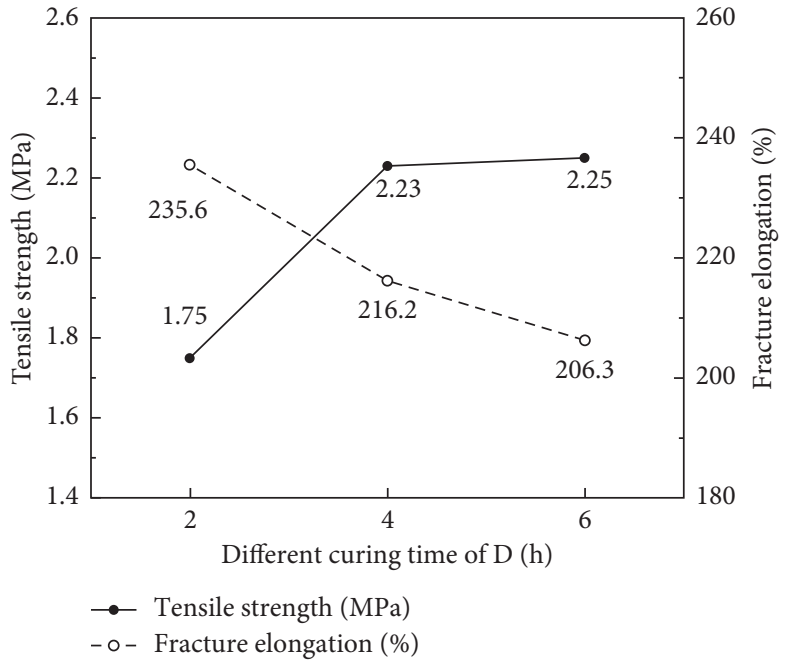

(b)

Figure 11: Tensile test results of UA (a) and DA (b) with different curing times.

Research studies on ERMABs show that when epoxy resin is used to modify the modified asphalt binder with this critical amount, it can play a more efficient role and contribute a tighter internal structure and better overall performance of the whole modified asphalt binders [26, 27]. Similar conclusions can be obtained from Figures 12(d), 12(e), and 12(f) of Figure 12.

Therefore, these photos of microscopic phase structures further verify the conclusion that the best ratio of epoxy resin to matrix asphalt binder in the modified system is 40 to 60.

4.2. TGA. TGA is an important method to study the thermal stability and composition of materials. In this section, TGA is used to study the differences in the thermal stability among ERMABs when the ratio of epoxy resin to matrix asphalt binder is $40: 60$.

Figure 13 shows TG and DTG test results of three types of ERMABs. It can be seen that their thermal decomposition processes are similar, with three stages. At the first stage, from room temperature to $250^{\circ} \mathrm{C}$, there is almost no thermal decomposition and mass loss is very small; thus, it is a thermally stable stage. The second stage, $250-550^{\circ} \mathrm{C}$, is the thermal decomposition stage, where the ERMABs exhibit slow mass loss for the first time in the range of $220-340^{\circ} \mathrm{C}$, caused by the volatilization of light-weight components of the ERMABs [28]. Then, there appears a sharp decline in thermal stability within the range of $340-450^{\circ} \mathrm{C}$, which is mainly caused by the decomposition of macromolecules and epoxy resin network within asphalt binders $[29,30]$. In addition, at this stage of rapid decline, 

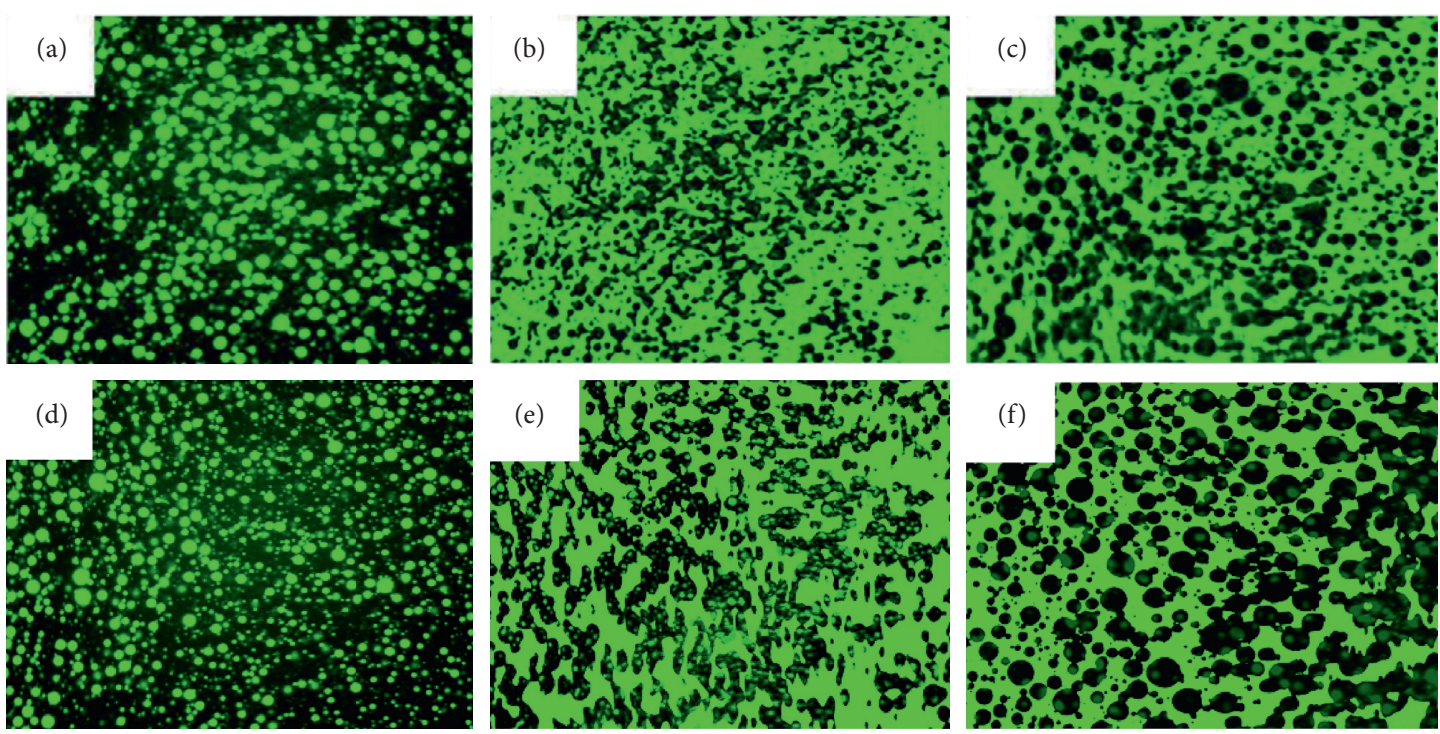

Figure 12: Fluorescent photos of binders with different dosages: (a) UA (30\% U); (b) UA (40\% U); (c) UA (50\% U); (d) DA (30\% D); (e) DA (40\% D); (f) DA (50\% (D).

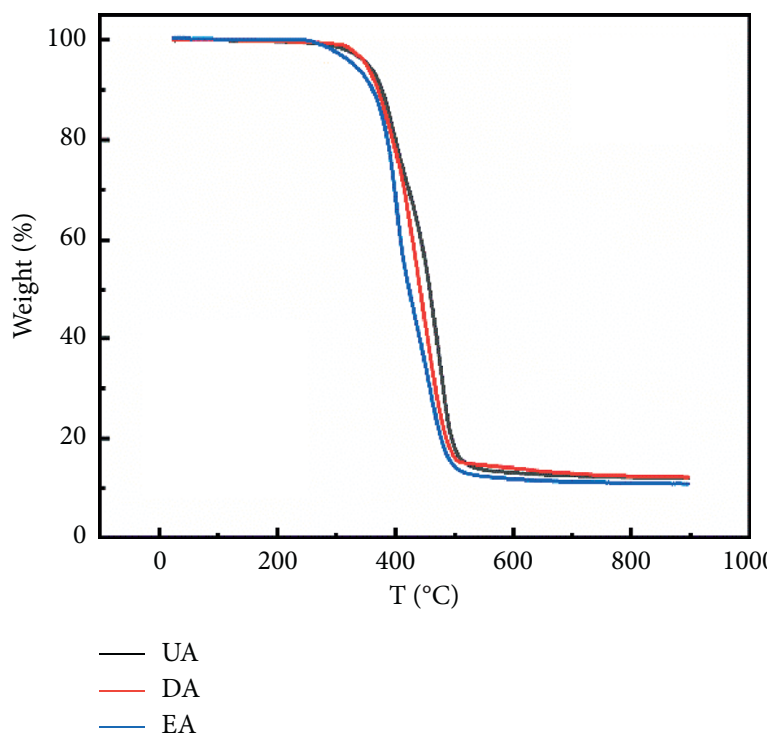

(a)

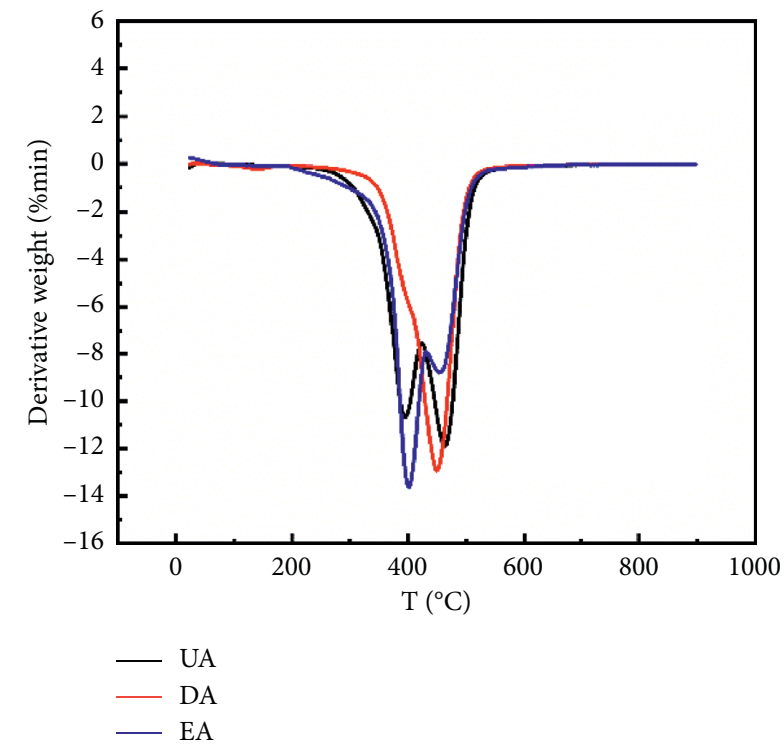

(b)

FIGURe 13: TG (a) and DTG (b) results of UA, DA, and EA.

the maximum decomposition temperature of EA is about $410^{\circ} \mathrm{C}$, while that of UA and DA is about $450^{\circ} \mathrm{C}$. The reason is that epoxy resins $U$ and $\mathrm{D}$ are modified by polyurethane and dimer acid, respectively. In the third stage, when the temperature exceeds $550^{\circ} \mathrm{C}$, the quality of the ERMABs basically remains unchanged. By calculating mass residue rates of ERMABs when the temperature is $800^{\circ} \mathrm{C}$, it can be known that the mass residue rate of $\mathrm{EA}$ is $11.04 \%$, while mass residue rate of the UA and DA is $13.21 \%$ and $13.76 \%$, respectively.

Based on the above analyses, it is concluded that the thermal stability of EA is the worst, while the thermal stability of UA and DA shows little difference.
4.3. Low-Temperature Performance. Improving the lowtemperature performance of ERMABs is one of the main purposes of this paper. Therefore, in this section, the low-temperature ductility test and low-temperature bending creep test are performed for the evaluation.

Figure 14 shows the low-temperature performance of three ERMABs. DA possesses the largest stretched length at either $5^{\circ} \mathrm{C}$ or $10^{\circ} \mathrm{C}$, followed by UA and EA. This indicates that DA has the best low-temperature performance, followed by UA and EA. Furthermore, in terms of the degree of reduction, no matter at $5^{\circ} \mathrm{C}$ or $10^{\circ} \mathrm{C}$, the tensile value of EA has a more significant drop compared with DA and UA. At $5^{\circ} \mathrm{C}$, the stretch value of EA decreased by $38.5 \%$ and $29.4 \%$ compared with DA 


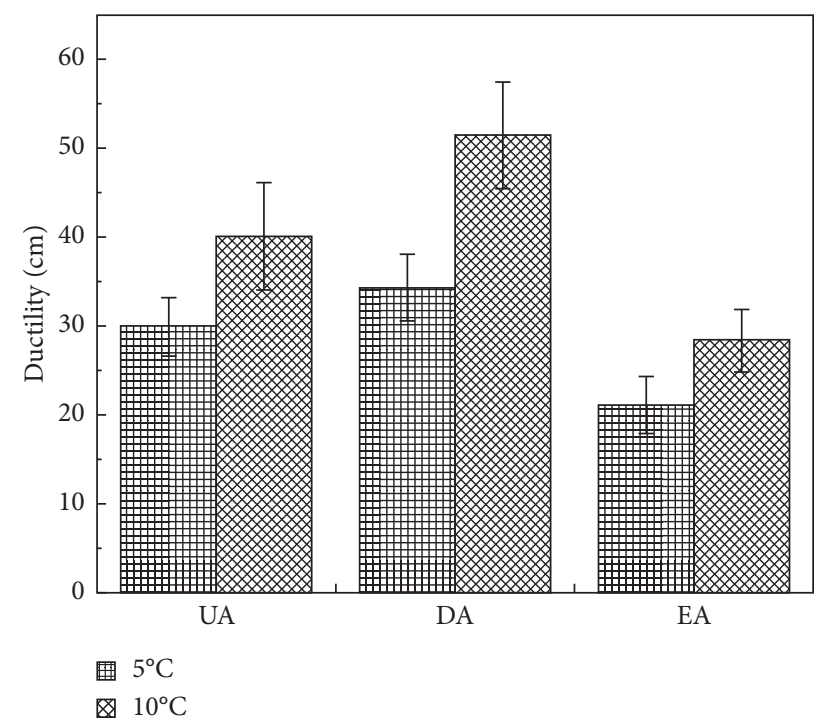

FIgure 14: Ductility test results of UA, DA, and EA at $5^{\circ} \mathrm{C}$ and $10^{\circ} \mathrm{C}$.

TABLE 2: BBR test results of UA, DA, and EA.

\begin{tabular}{|c|c|c|c|c|c|c|c|c|}
\hline \multirow{2}{*}{ Types of binders } & \multicolumn{2}{|c|}{$-6^{\circ} \mathrm{C}$} & \multicolumn{2}{|c|}{$-12^{\circ} \mathrm{C}$} & \multicolumn{2}{|c|}{$-18^{\circ} \mathrm{C}$} & \multicolumn{2}{|c|}{$-24^{\circ} \mathrm{C}$} \\
\hline & $\mathrm{S}(\mathrm{MPa})$ & $\mathrm{m}$ & $\mathrm{S}(\mathrm{MPa})$ & $\mathrm{m}$ & $\mathrm{S}(\mathrm{MPa})$ & $\mathrm{m}$ & $\mathrm{S}(\mathrm{MPa})$ & $\mathrm{m}$ \\
\hline UA & 95 & 0.443 & 177 & 0.349 & 282 & 0.311 & 526 & 0.209 \\
\hline DA & - & - & - & - & 205 & 0.358 & 305 & 0.307 \\
\hline EA & 201 & 0.366 & 298 & 0.297 & 497 & 0.253 & - & - \\
\hline
\end{tabular}

and UA, respectively; at $10^{\circ} \mathrm{C}$, the stretch value of EA decreased by $45.0 \%$ and $28.7 \%$ compared with DA and UA, respectively. This result indicates that the low-temperature performance of EA may be far worse than the other two modified asphalt binders prepared in this experiment.

Table 2 shows the creep stiffness modulus (S) and stress relaxation speed $(\mathrm{m})$ of three types of ERMABs at different test temperatures. According to Table 2, as the temperature decreases, $\mathrm{S}$ increases rapidly and $\mathrm{m}$ decreases significantly, which means the low-temperature performance of the binders gets worse. SHRP research achievements reveal that the large creep stiffness modulus and the small relaxation speed make the pavement easy to crack at low temperatures. It is believed that the test binder could be used in the $10^{\circ} \mathrm{C}$ lower than the test temperature, where the $S$ value of test binder is no larger than $300 \mathrm{MPa}$ and $\mathrm{m}$ value is no smaller than 0.3 under the test procedure of ASTM D6373 [21]. According to this proposal, when the temperature is lower than $-22^{\circ} \mathrm{C}$, EA cannot be used, while UA and DA can still be used at $-28^{\circ} \mathrm{C}$. Moreover, the low-temperature limit of DA is even close to $-34^{\circ} \mathrm{C}$. These analyses reveal that the epoxy resin modified by polyurethane and dimer acid can greatly improve the low-temperature performance of the modified asphalt binders, showing the significance of the method on improving the low-temperature performance of modifiers.

The ductility and BBR test results both show that the modified asphalt binders by $U$ and $D$ could significantly improve the low-temperature performance of ERMAB.
4.4. Temperature Dependence. ERMAB still belongs to temperature-sensitive material dominated by asphalt binder, and its rheological properties would also change along with the test temperature. Figure 15 shows the complex moduli and phase angles of ERMABs within the temperature range of $30-90^{\circ} \mathrm{C}$.

As the temperature increases, all the complex moduli decrease and phase angles increase, indicating that temperature increase will significantly reduce the deformation resistance of ERMABs. By comparing the test results of three types of ERMABs, the following conclusions can be drawn. First of all, EA, with the largest complex modulus and smallest phase angle, possesses the best resistance to deformation within the medium- and high-temperature ranges. Secondly, the complex modulus of UA is slightly lower than that of EA on the whole, but there is little difference between the two within the temperature range of $40-65^{\circ} \mathrm{C}$. This shows that although the deformation resistance of UA is slightly lower than that of EA in the middle and high-temperature range, there is little difference in performance between the two in some certain temperature range. Thirdly, compared with the above two, DA has smaller complex modulus and larger phase angles. However, within the testing temperature range, the rate of decrease of its complex modulus is lower, making it finally closer to the complex moduli of EA and UA at relatively high-temperature stage. This indicates that DA has the worse temperature resistance to deformation in the testing temperature range, but its sensitivity to temperature changes is the lowest. 


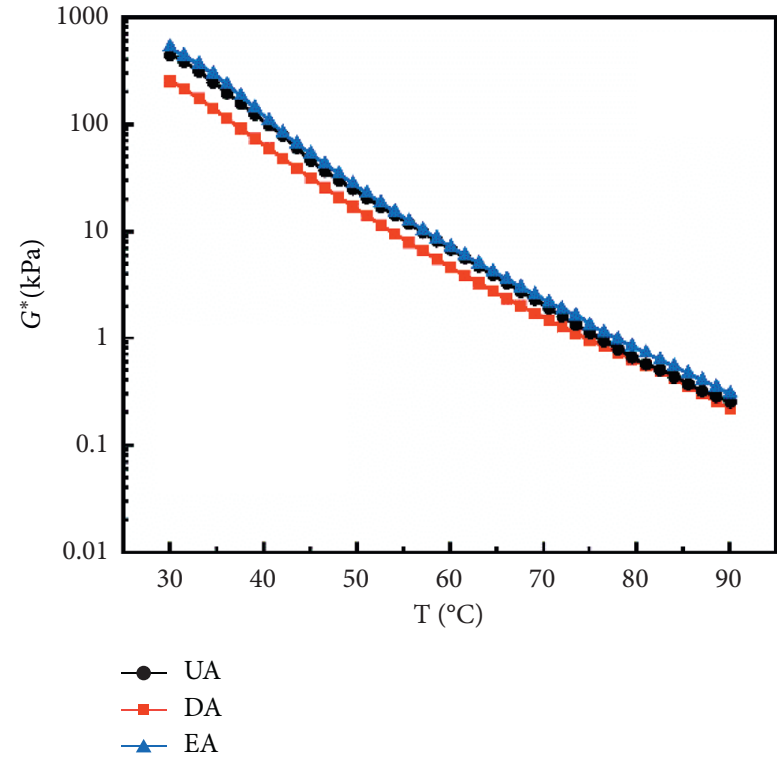

(a)

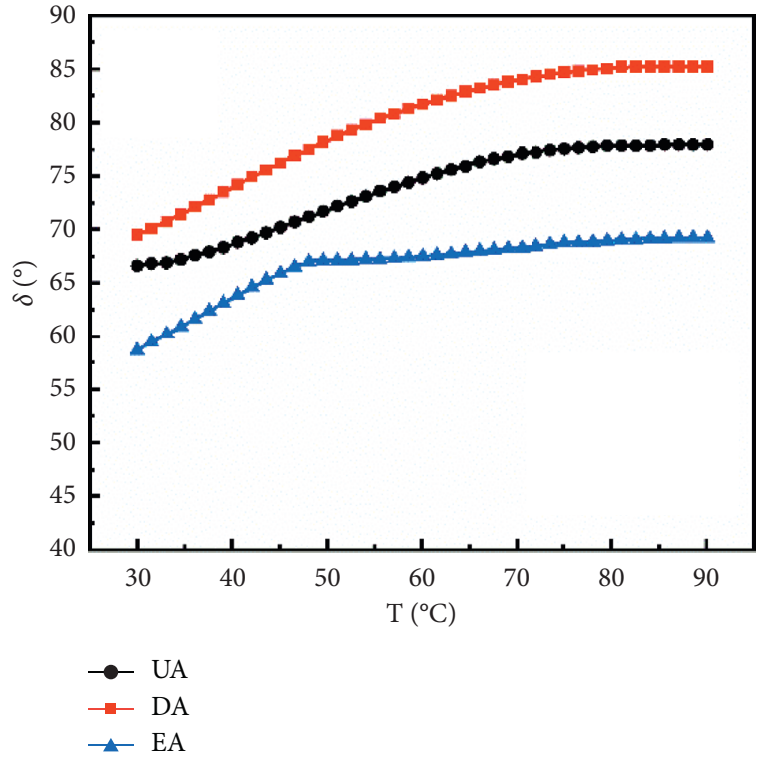

(b)

FIgURE 15: Complex moduli (a) and phase angles (b) of UA, DA, and EA.

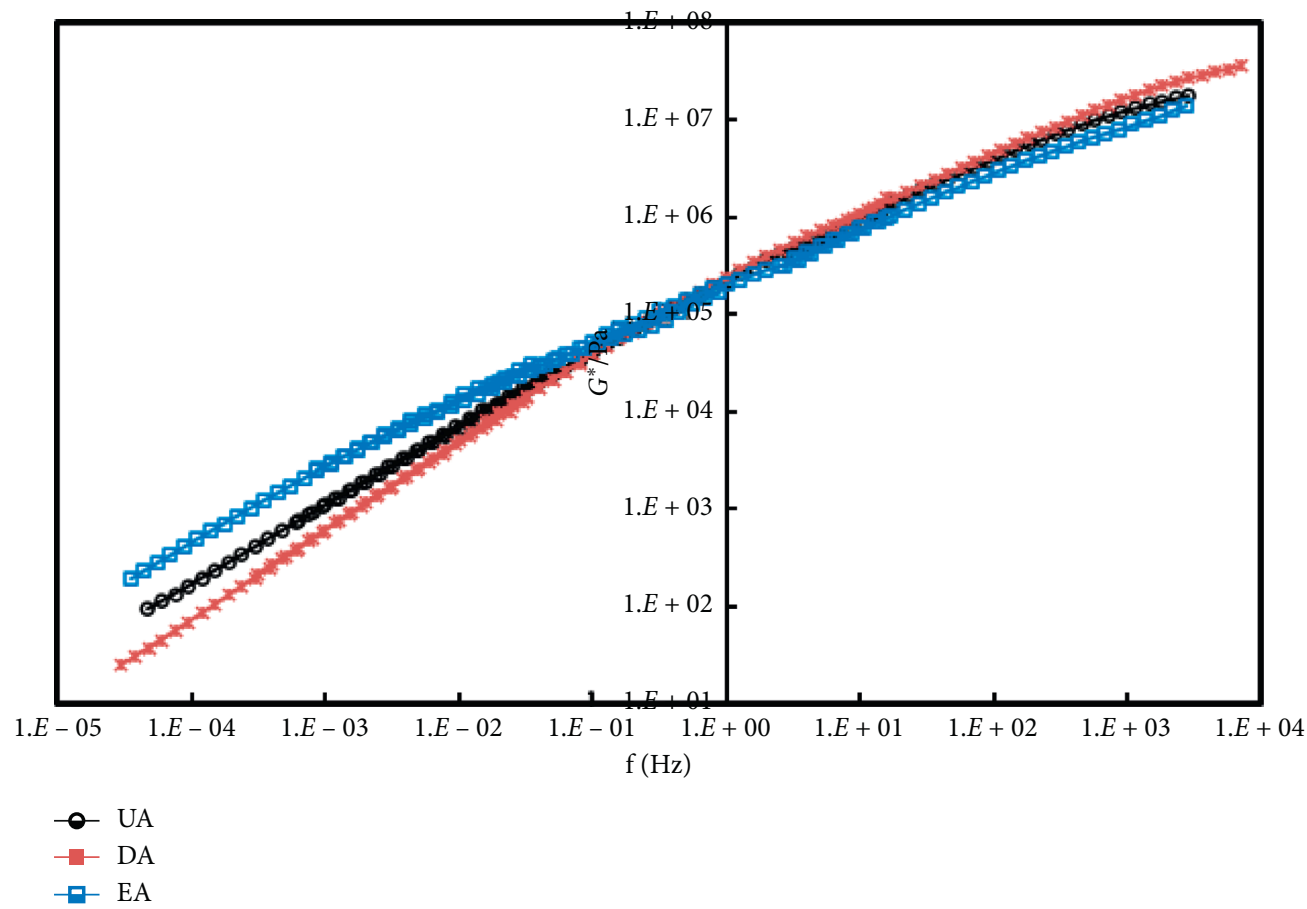

FIgURE 16: Complex modulus master curves of UA, DA, and EA.

In summary, EA still has a slight advantage in resisting temperature deformation within the medium- and hightemperature ranges, while DA has lower temperature sensitivity in the entire temperature range.

4.5. Frequency Dependence. In this section, the frequency sweep test is performed to study the frequency sensitivity of the dynamic shear modulus of ERMABs within the range of linear viscoelasticity.

Figure 16 shows master curves of complex moduli of ERMABs. As the frequency increases, the complex moduli are significantly increased, showing a clear dependence on frequency. Specifically, in the low frequency $\left(10^{-5}-10^{-1} \mathrm{~Hz}\right)$, EA has the highest complex modulus, indicating that it possesses the best high-temperature stability. However, as 


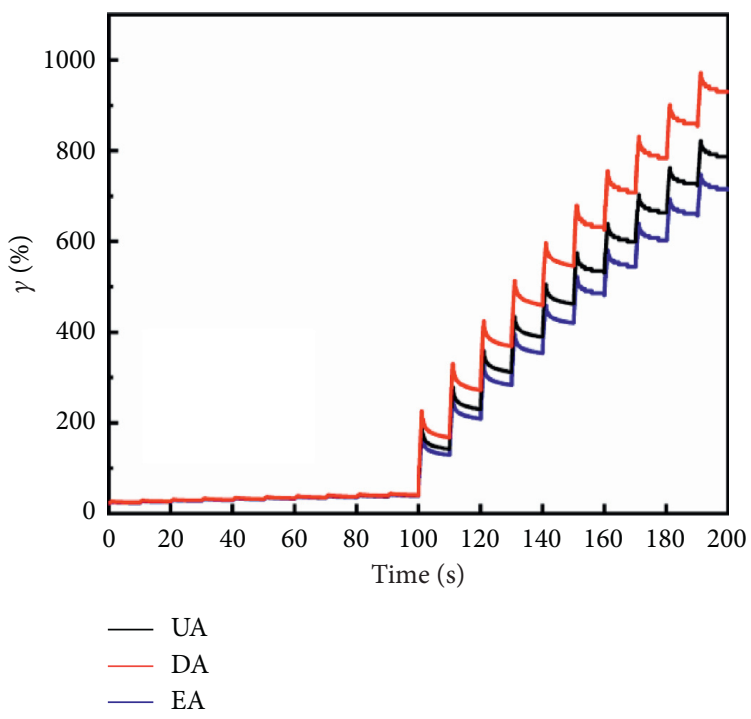

FIgURE 17: Results of shear strain-cycles of UA, DA, and EA in the MSCR test.

TABLE 3: Results of $\mathrm{J}_{\mathrm{nr}}$ and $\mathrm{R}$ in the MSCR test.

\begin{tabular}{lcccc}
\hline & & UA & DA & EA \\
\hline \multirow{2}{*}{$\mathrm{R}(\%)$} & $0.1 \mathrm{kPa}$ & 60.90 & 58.70 & 61.87 \\
& $3.2 \mathrm{kPa}$ & 34.04 & 32.21 & 36.83 \\
\hline \multirow{2}{*}{$\mathrm{Jnr} \times 10^{-3}$} & $0.1 \mathrm{kPa}$ & 20 & 26 & 17 \\
& $3.2 \mathrm{kPa}$ & 25 & 29 & 21 \\
\hline
\end{tabular}

the frequency continues to increase, the growth rate of curve EA decreases and approaches to curves DA and UA within the medium frequency $\left(10^{-1}-10^{1} \mathrm{~Hz}\right)$ and then gradually drops to be the lowest within the subsequent high frequency $\left(10^{1}-10^{4} \mathrm{~Hz}\right)$. This shows that there is little difference in the shearing resistance performance of the three at the medium temperature stage, while at the low-temperature stage, the cracking resistance performance of EA is the worst. This is consistent with the conclusion gained in the previous BBR test and temperature sweep test. Based on three frequency master curves, it can be seen that EA has the lowest dependence to frequency, followed by UA and DA.

4.6. Fatigue Performance. In this section, the fatigue performance of ERMABs is evaluated by MSCR. Figure 17 shows the cycle-strain test results. It can be known that three types of ERMABs show no obvious deformation when the shear stress is $0.1 \mathrm{kPa}$. However, once the shear stress increased to $3.2 \mathrm{kPa}$, all shear strain increased sharply, and significant cumulative deformation appears. Specifically, the shear strain and cumulative deformation of EA are the smallest, followed closely by UA, which gradually appears differences in shear strain and cumulative deformation along with the test cycles. In terms of DA, it emerges much larger shear strain and cumulative deformation since it is under the $3.2 \mathrm{kPa}$ shearing stress, and the cumulative deformation is nearly $40 \%$ higher than that EA after the test. So, it can be known that DA has the worst fatigue performance.
Furthermore, the recovery rate $(\mathrm{R})$ and nonrecoverable recovery creep compliance (Jnr) of ERMABs in the deformation are calculated and are shown in Table 3. It can be known that when the shear stress is $0.1 \mathrm{kPa}$, the deformation recovery rate and nonrecoverable creep compliance of all binders are very similar; while when the shear stress is $3.2 \mathrm{kPa}$, the deformation recovery rate of $\mathrm{UA}$ and $\mathrm{DA}$ is $92.4 \%$ and $87.5 \%$ of $\mathrm{EA}$, and nonrecoverable creep compliance is $119.0 \%$ and $138.1 \%$ of EA, respectively. This result further illustrates that the fatigue resistance of UA and DA is worse than that of the control sample.

\section{Conclusions}

This paper tries to improve the low-temperature performance of ERMABs with not extended curing time. Three modified epoxy resins were prepared to modify the A-70 matrix asphalt binder. Then, the formula and prepared methods of ERMAB were optimized and determined. Moreover, a comparative analysis on the overall performance between two prepared ERMABs and the control sample were carried out. Main conclusions could be drawn as follows:

(1) Polyurethane-modified epoxy resin or dimer acidmodified epoxy resin, with a suitable curing agent, can significantly improve the low-temperature performance of ERMAB, and the curing time meets the construction requirements. The optimal ratio of $U$ to matrix asphalt binder to $\mathrm{P}$ is $40: 60: 4.4$, and the optimal ratio of $\mathrm{O}$ to matrix asphalt binder to $\mathrm{P}$ is 40 : $60: 1.9$ by weight. The curing temperature is $120^{\circ} \mathrm{C}$, and the curing time is $4 \mathrm{~h}$.

(2) When the ratio of epoxy resin to A-70 was $40: 60$, ERMAB witnesses the reversal of continuous phases and the best overall performance; thus, this ratio is appropriate.

(3) The low-temperature performance of two ERMABs is significantly improved, and the application 
temperature can even extend $18^{\circ} \mathrm{C}$. Compared with the control sample, two ERMABs have almost the same rheological properties at medium temperature but slightly reduced high-temperature performance and fatigue resistance.

(4) The epoxy resin asphalt binder modified by polyurethane possesses the best overall performance. Compared with the control sample, its low limit service temperature can extend $12^{\circ} \mathrm{C}$, while its rheological property and fatigue resistance at mediumand high-temperature ranges show very little deterioration. Thus, it is an ideal and practical ERMAB with improved low-temperature performance.

\section{Data Availability}

The data used to support the findings of this study are available from the corresponding author upon request.

\section{Conflicts of Interest}

The authors declare no conflicts of interest.

\section{Acknowledgments}

This work was supported by the National Natural Science Foundation of China (no. 52008042) and Open Fund of Key Laboratory of Special Environment Road Engineering of Hunan Province (Changsha University of Science and Technology, grant no. kfj190502), and the authors gratefully acknowledge their financial support.

\section{References}

[1] X. Wang, R. Wu, and L. Zhang, "Development and performance evaluation of epoxy asphalt concrete modified with glass fibre," Road Materials and Pavement Design, vol. 20, no. 3, pp. 715-726, 2019.

[2] X. Shi, H. Zhang, X. Bu, G. Zhang, H. Zhang, and H. Kang, "Performance evaluation of BDM/unsaturated polyester resin-modified asphalt mixture for application in bridge deck pavement," Road Materials and Pavement Design, vol. 22, pp. 1-17, 2020.

[3] Q. Lu and J. Bors, "Alternate uses of epoxy asphalt on bridge decks and roadways," Construction and Building Materials, vol. 78, pp. 18-25, 2015.

[4] P. Cong, J. Yu, and S. Chen, "Effects of epoxy resin contents on the rheological properties of epoxy-asphalt blends," Journal of Applied Polymer Science, vol. 118, no. 6, pp. 3678-3684, 2010.

[5] J. Yu, P. Cong, and S. Wu, "Laboratory investigation of the properties of asphalt modified with epoxy resin," Journal of Applied Polymer Science, vol. 113, no. 6, pp. 3557-3563, 2009.

[6] Y. Kang, M. Song, L. Pu, and T. Liu, "Rheological behaviors of epoxy asphalt binder in comparison of base asphalt binder and SBS modified asphalt binder," Construction and Building Materials, vol. 76, pp. 343-350, 2015.

[7] H. Yin, Y. Zhang, Y. Sun et al., "Performance of hot mix epoxy asphalt binder and its concrete," Materials and Structures, vol. 48, no. 11, pp. 3825-3835, 2015.
[8] P. Cong, W. Luo, P. Xu, and Y. Zhang, "Chemical and physical properties of hot mixing epoxy asphalt binders," Construction and Building Materials, vol. 198, pp. 1-9, 2019.

[9] J. Gong, Y. Liu, Q. Wang et al., "Performance evaluation of warm mix asphalt additive modified epoxy asphalt rubbers," Construction and Building Materials, vol. 204, pp. 288-295, 2019.

[10] Y. Jiang, X. Han, J. Gong et al., "Laboratory investigation of epoxy asphalt binder modified by brominated SBS," Construction and Building Materials, vol. 228, Article ID 116733, 2019.

[11] J. Xin, M. Li, R. Li, M. P. Wolcott, and J. Zhang, "Green epoxy resin system based on lignin and tung oil and its application in epoxy asphalt," ACS Sustainable Chemistry \& Engineering, vol. 4, no. 5, pp. 2754-2761, 2016.

[12] Y. Xue and Z. Qian, "Development and performance evaluation of epoxy asphalt concrete modified with mineral fiber," Construction and Building Materials, vol. 102, pp. 378-383, 2016.

[13] J. Zhang, W. Su, Y. Liu et al., "Laboratory investigation on the microstructure and performance of SBS modified epoxy asphalt binder," Construction and Building Materials, vol. 270, Article ID 121378, 2020.

[14] R. Liang, R. Jin, D. Zhou, W. Sun, and Y. Kang, "Nonlinear rheological behaviors of epoxy asphalt binder compared to base asphalt binder and SBS modified asphalt binder at above ambient temperatures," Construction and Building Materials, vol. 250, Article ID 118755, 2020.

[15] P. Cong, S. Chen, J. Yu, and H. Chen, "Compatibility and mechanical properties of epoxy resin modified asphalt binders," International Journal of Pavement Research and Technology, vol. 4, no. 2, p. 118, 2011.

[16] Y. Jiang, Y. Liu, J. Gong et al., "Microstructures, thermal and mechanical properties of epoxy asphalt binder modified by SBS containing various styrene-butadiene structures," $\mathrm{Ma}$ terials and Structures, vol. 51, no. 4, pp. 1-13, 2018.

[17] C. Hu, J. Zhao, Z. Leng, M. N. Partl, and R. Li, "Laboratory evaluation of waterborne epoxy bitumen emulsion for pavement preventative maintenance application," Construction and Building Materials, vol. 197, pp. 220-227, 2019.

[18] ASTM, "Viscosity of epoxy resins and related components, D2393," ASTM International, West Conshohocken, PA, USA, 1986.

[19] ASTM, Standard Test Method for Tensile Properties of Plastics, D638, ASTM International, West Conshohocken, PA, USA, 2014.

[20] ASTM, Standard Test Method for Ductility of Asphalt Materials, D113, ASTM International, West Conshohocken, PA, USA, 2017.

[21] ASTM, Standard Specification for Performance Graded Asphalt Binder, d6373, ASTM International, West Conshohocken, PA, USA, 2016.

[22] American Association of State Highway and Transportation Officials, "Performance-Graded Asphalt Binder Using Multiple Stress Creep Recovery (Mscr) Test," American Association of State Highway and Transportation Officials MP19, Washington, D.C USA, 2010.

[23] S. Tsutsumi, Y. Kato, K. Namba, and H. Yamamoto, "Functional composite material design using Hansen solubility parameters," Results in Materials, vol. 4, Article ID 100046, 2019.

[24] K. Keshmiri, H. Huang, and N. Nazemifard, "Compatibility of poly (dimethylsiloxane) microfluidic systems with high 
viscosity hydrocarbons," SN Applied Sciences, vol. 1, no. 7, pp. 1-11, 2019.

[25] JTG/T3364-02-2019, Specifications For Design and Construction of Pavement on Highway Steel Deck Bridge, People's Communications Publishing Co., Ltd, Beijing, China, 2019, in Chinese.

[26] J. Jin, T. Xiao, J. Zheng et al., "Preparation and thermal properties of encapsulated ceramsite-supported phase change materials used in asphalt pavements," Construction and Building Materials, vol. 190, pp. 235-245, 2018.

[27] B. Ma, X.-Q. Wang, X.-Y. Zhou, K. Wei, and W. Huang, "Measurement and analysis of thermophysical parameters of the epoxy resin composites shape-stabilized phase change material," Construction and Building Materials, vol. 223, pp. 368-376, 2019.

[28] M. K. Pitchan, S. Bhowmik, M. Balachandran, and M. Abraham, "Effect of surface functionalization on mechanical properties and decomposition kinetics of high performance polyetherimide/MWCNT nano composites," Composites Part A: Applied Science and Manufacturing, vol. 90, pp. 147-160, 2016.

[29] Y. Zhang, Y. Sun, K. Xu et al., "Brucite modified epoxy mortar binders: flame retardancy, thermal and mechanical characterization," Construction and Building Materials, vol. 93, pp. 1089-1096, 2015.

[30] H. Wu, V. K. Thakur, and M. R. Kessler, "Novel low-cost hybrid composites from asphaltene/SBS tri-block copolymer with improved thermal and mechanical properties," Journal of Materials Science, vol. 51, no. 5, pp. 2394-2403, 2016. 